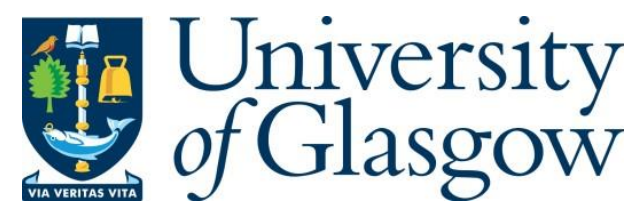

Kamphorst, J. J., and Lewis, I. A. (2017) Editorial overview: recent innovations in the metabolomics revolution. Current Opinion in Biotechnology, 43, iv-vii.

There may be differences between this version and the published version. You are advised to consult the publisher's version if you wish to cite from it.

http://eprints.gla.ac.uk/136336/

Deposited on: 14 March 2017

Enlighten - Research publications by members of the University of Glasgow http://eprints.gla.ac.uk 


\section{Editorial overview: Recent innovations in the metabolomics revolution}

Jurre J Kamphorst and Ian A Lewis

Current Opinion in Biotechnology 2017, 43:iv-vii For a complete overview see the Issue Available online 23rd January 2017 http://dx.doi.org/10.1016/j.copbio.2017.01.005 0958-1669/ã 2017

Elsevier Ltd. All rights reserved.

Metabolism involves a complex set of chemical processes that allow organisms to transform nutrients into energy, reducing power, and the diverse range of cellular building blocks necessary for life. Although metabolism has been intensively studied for more than a century, the technology for understanding metabolic phenomena from a comprehensive, network-level perspective has only been available for a short time. The metabolomics approach - the analysis of all observable metabolites in complex biological samples - has rapidly advanced with the introduction of highresolution mass spectrometry, sophisticated chromatography, multidimensional nuclear magnetic resonance spectroscopy, clever isotope labeling strategies, and powerful software. These technological developments have dominated the field over the last two decades and have laid the foundation for the recent explosion in demand for metabolomics. In this special edition of Current Opinion in Biotechnology, we have invited a selection of both well established and emerging leaders of the metabolomics field to describe the current state-of-the-art as well as their visions for the future of metabolomics. These authors represent a cross-section of researchers who are driving the modern renaissance of metabolism research through technical innovation and biological creativity.

\section{Mass spectrometry}

Mass spectrometry (MS) has become the most widely used analytical platform in metabolomics because of its high sensitivity, excellent dynamic range, and ability to analyze a wide range of molecules. Although metabolomics studies strive to analyze theoretically all metabolites, capturing molecules with vastly different chemical properties is impossible using traditional chromatography. In this special issue, Burgess and Haggarty discuss recent innovations in liquid and gas chromatography that are taking metabolomics a step closer to global coverage of the metabolome. They highlight the benefits of placing two separation columns in series, column switching, and various approaches to two-dimensional chromatography mass spectrometry (2D LC-MS) that capture a much broader range of compounds than is currently possible. Recent developments in supercritical fluid chromatography - a technique that has yet to reach its full potential in metabolomics - are also discussed in this review.

Another technology that may dramatically increase metabolome coverage is capillary electrophoresis (CE). CE is an exciting alternative to more traditional liquid and gas chromatography. It has unrivaled separation efficiency and the ability to cope with low sample volumes. Unfortunately, hyphenating CE with mass spectrometry has proven to be a major technical challenge. Ramautar et al. describe recent innovations in interfacing CE to MS using a novel sheathless porous tip interface. These innovations are playing a critical role in improving the performance and practicality of CE-MS and may eventually bring this transformative technology to the forefront of metabolomics. 
Another emerging frontier of metabolomics is MS-based tissue imaging, a challenging application of metabolomics with a tremendous scientific potential. Borchers et al. are pioneering new approaches in matrix-assisted laser desorption/ionization mass spectrometry (MALDI-MS), including new types of matrices and new ways to deposit them. These technical advances have made it possible to study a wider range of proteins, peptides, lipids, drug molecules, and metabolites than what was previously possible.

Lipidomics, the comprehensive characterization of the lipid component of biological systems, is a rapidly expanding sub-discipline of metabolomics. As Tumanov and Kamphorst argue, the widespread characterization of lipids as homogenous apolar molecules belies their true structural diversity. They describe a practical combination of extraction and separation procedures that dramatically improves the scope and chemical diversity of molecules that can be captured in lipidomic analyses, and describe some of the powerful new applications of this emerging approach.

\section{Nuclear magnetic resonance spectroscopy}

Nuclear magnetic resonance (NMR) spectroscopy played a pivotal role in the early development of metabolomics and continues to provide an essential orthogonal analytical approach to MS. NMR's outstanding quantitative performance, ability to detect any organic molecule in solution within its sensitivity limit, and the ability to unambiguously characterize novel compounds de novo ensures that NMR will retain an important place in field. However, the increasing popularity of MS-based approaches has encouraged the leaders of the NMR-based metabolomics community to critically evaluate the evolving role of NMR. The future vision, as articulated by seven preeminent North American NMR spectroscopists - John L Markley, Rafael Brüschweiler, Arthur S Edison, Hamid R Eghbalnia, Robert Powers, Daniel Raftery, and David S Wishart - is an exciting contribution to this special issue. In addition to this long-term vision, this special issue includes several focused contributions from the frontiers of NMR-based technology. Giraudeau et al. describe several recent breakthroughs in multidimensional NMR techniques, high sensitivity analyses, and high-throughput approaches that collectively represent the future for NMR-based studies. Likewise, Brüschweiler and Bingol introduce a powerful suite of computational, chemical, and analytical techniques that dramatically improve the scope of NMR-based approaches and extend the number of molecules that can be robustly identified in a single assay.

\section{Computation/bioinformatics}

Ongoing technical innovations in metabolomics are driving a continual increase in the size and complexity of metabolomics datasets. Analyzing these data and preserving a usable record of each study is a significant challenge. Commercial software tools, such as those distributed by instrument manufactures, have been unable to keep pace with the rapid advances in the field. Consequently, a worldwide network of academic groups has been driving the development of software packages, data standards, and open source databases for metabolomics.

In this special edition, Eghbalnia et al. examine the current state of public data archive efforts and articulate the need for a robust data standard. Although their article is written through the lens of 
NMR-based studies, establishing robust data standards and comprehensive data archival systems is critical for all analytical approaches to metabolomics. In a related article, Sarpe and Schriemer discuss how a modular coding strategy could significantly improve the portability and potency of academic software development efforts. Rather than building software solutions from scratch, this modular approach would allow existing algorithms and workflows to be easily integrated into novel software tools.

The recent advances in data standards, public databases, and modular software development are taking the field a step closer towards fully automated data analyses. In this issue, Hamzeiy and Cox examine the potential avenues through which proteomics informatics approaches could be repurposed for automating untargeted analyses of small molecules. They argue that the non-linear mass recalibration algorithm used by MaxQuant could greatly improve the accuracy of metabolite assignments. Similar efforts are also underway in the lipidomics community. Wakelam et al. examine the current state of lipidomics oriented bioinformatics and articulate the need for new software approaches that can integrate lipid assignments into meaningful datasets reflecting lipid pathway activity.

\section{Innovative applications}

The maturation of metabolomics technology has stimulated a world-wide boom in metabolomics applications in diverse fields ranging from food science to cancer biology. This special edition includes a small sampling of some of the new avenues through which metabolomics is driving improved understanding of the natural world.

One of the most powerful recent applications of metabolomics has been the engineering of microorganisms to facilitate the production of biofuels. Finding effective methods for producing biofuels is a global priority and microbes are key to making these alternative fuels economically feasible. Reviews by the Antoniewicz and Amador-Noguez groups describe the important role metabolomics is playing in the optimization of microbial biofuel production from plant-based lignocellulosic biomass (which contains cellulose, hemicellulose, and lignin). For example, Amador-Noguez and Martien discuss how comprehensive metabolomics studies have identified previously unanticipated mechanisms of product inhibition in microbes, and how pretreatment of lignocellulosic biomass can affect the ability of microbes to produce biofuels. Antoniewicz and Gonzalez's article describes how stable isotope tracing has been used to guide engineering of efficient biofuel producing strains. These fluxomics approaches - the comprehensive analysis of carbon flow through complex metabolic networks - are further detailed in an article by Létisse et al. They describe the current state-of-the-art, high-throughput stable isotope tracing methods, mathematical modeling, and how this powerful technology could be adapted to support a wide range of applications.

Another exciting example of novel metabolomics applications is provided by Weljie et al. They describe the emerging role metabolomics is playing in our understanding of circadian rhythms and the surprising connections these rhythms have on metabolism. They detail the recent studies linking medium-chain acyl-carnitines, lysophosphatidylcholines, and phosphatidylcholines levels to circadian rhythm and provide some guidelines for harnessing metabolomics technology in future studies. 
This special issue includes several articles related to emerging analytical approaches for investigating particular classes of compounds. An article by Nakabayashi and Saito, for example, discuss how advances in high resolution mass spectrometry have created an opportunity for comprehensive analysis of sulfur containing molecules (S-metabolites). Sulfur is an essential element that is present in a diverse range of metabolites and this new analytical approach may offer a mechanism for investigating this often overlooked element. The authors draw particular attention to plant-based applications, where sulfur-related analyses could play an important role in novel compound identification. The theme of plant metabolism is continued by Freund and Hegeman, who review stable isotope labelling approaches in plants. The authors explain how these approaches aid in plant metabolite annotation, quantification, and pathway elucidation.

Digestomics, the comprehensive analysis of protein catabolism, is another expanding sub-discipline that exists in a largely unexplored space between proteomics and metabolomics. Bingeman et al. introduce this concept, discuss the challenges and opportunities emerging from this new discipline, and provide some practical guidelines about how to conduct experiments using this exciting new analytical approach. This hybrid proteomics-metabolomics theme is continued in an article by Nomura et al., who discuss how enzymatic activity can be directly assessed in a proteome-wide setting using activity-based protein profiling (ABPP). This approach uses active site-directed or reactivity-based chemical probes to determine enzymatic activity in complex mixtures. They describe successful applications of ABPP, including the discovery that aggressive cancers express certain lipases. This emerging approach has many exciting applications, including helping to identify new disease relevant targets, assisting in the development of small molecule inhibitors, and providing a platform for assessing vi Analytical biotechnology Current Opinion in Biotechnology 2017, 43:iv-vii www.sciencedirect.com proteome-wide selectivity and off-targets of chemical therapies.

In summary, this special issue of Current Opinion in Biotechnology provides a cross-section of the current state-of-the art in metabolomics technology and highlights a few of the many applications of this emerging technology. These modern metabolomics approaches are radically expanding the scope of routine studies and driving a renaissance in metabolism research. Editorial overview Kamphorst and Lewis vii www.sciencedirect.com Current Opinion in Biotechnology 2017, 43:iv-vii 